Japan. J. Genetics Vol. 55, No. 3: 151-164 (1980)

\title{
ISOZYME POLYMORPHISM IN SPECIES OF THE GENUS VICIA (LEGUMINOSAE)
}

\author{
KIYOSHI YAMAMOTO AND UZI PLITMANN* \\ Faculty of Agriculture, Kagawa University, Miki-cho, Kagawa-ken 461-07, and \\ Department of Botany, The Hebrew University, Jerusalem, Israel*
}

Received December 28, 1979

Thirty nine strains of 24 Vicia species representing the main sections of the genus, have been analyzed for their isozyme patterns by disc electrophoresis of leaf and cotyledon extracts. The enzyme systems studied were amylase, esterase, glutamate oxaloacetic transaminase (GOT), indo-phenol oxidase (IPO).

Comparing the zymograms obtained, one can arrive at the following conclusions: There are many bands common to species, series of sections and, in contrast, there are some bands differentiating between certain taxonomic categories. Such characterizing bands and patterns do not feature any clear general phylogenetic trend or systematic correlation. The enzymatic similarity does not necessarily correspond with the taxonomic relationships. GOT show lesser polymorphism, compared with amylase and esterase. The latter systems might be helpful in identifying species or strains.

Species which are polymorphic in many other characters reveal also enzymatic polymorphism. Other possible correlations are briefly discussed, with a special note on the evolutionary implications of such a study in Vicia.

\section{INTRODUCTION}

The genus Vicia contains over 130 species, distributed in the temperate zones of both hemispheres but centered mainly in the Mediterranean region, Western Asia and Western South America. The genus has never been monographed on a world basis. Kupicha (1976) restricted its number of species to about 140; some other consider the number to be even higher. About 10 species are of economic importance as grain or forage crops. The species are grouped into six sections (Plitmann 1970) and as many as 15 series.

The genus is rather diverse in many respects: Habitats, habitus, morphology of the reproductive organs, and karyotypes. Certain species, or groups, feature wide morphological variation as well as tremendous karyotype polymorphism (Hollings and 
Stace 1974; Ladizinsky 1978; Zohary and Plitmann 1979). One of the authors (Yamamoto 1973) has studied the karyotypes of several species and re-discussed the relationships between karyotypes and other characters.

Our study of isozyme patterns in Vicia has been aimed to clarify or confirm some systematic relationships in the genus. It was likewise of interest to find out correlations between isozyme patterns and certain taxonomic criteria and to estimate their taxonomic value. Such kind of work has been carried out with few other genera, composed of both cultivated and wild species, as Tomatoes (Rick and Fobes 1975) and Brassica (Phelan and Vaughan 1976).

Besides, it may have bearings on the general problem of selective versus neutral isoenzymes as presented by Endo (1973), Johnson (1973, 1975), Zouros (1975) and many others.

\section{MATERIALS AND METHODS}

Thirty nine strains of 24 Vicia taxa, annuals and perennials, representing the four main sections, were used for isozyme analysis of leaves. Unfortunately, we got only very few seeds of the New World species. The species investigated, seed sources and origins are listed in Table 1. The seeds (some of which were actually second generation) were grown in experimental plots at the Faculty of Agriculture of Kagawa University.

Isozyme detection was made by the polyacrylamide disc electrophoretic method (Davis 1964). Staining methods were applied from Brewer (1970). The leaves used for examination were taken from 4 th to 7 th nodes. This leaf age was suitable for stable isozyme band patterns in this genus as reported previously (Yamamoto 1975).

Leaf extracts were made by grinding a mixture of 2 grams of leaflets with the same weight of $0.05 \mathrm{M}$ Tris- $\mathrm{HCl}$ buffer solution $(\mathrm{pH}=7.54)$. To each column, 0.1-0.04 $\mathrm{ml}$ of sap were applied. Isozyme band patterns of amylase, esterase, glutamate oxaloacetic transaminase (GOT) and indo-phenol oxidase (IPO) were detected in all strains. Substrate and staining dyes used for each enzyme system are detailed in Table 2. $\alpha$ - and $\beta$-amylase isozyme bands were determined according to the method of Frydenberg and Nielsen (1965) and Yamamoto (1975).

Cotyledon (seedlings) extracts were used in previous preliminary experiments. Besides the 4 aforementioned enzyme systems, $\mathrm{ADH}$ and peroxidase (electrofocusing) were then also examined. Banding patterns in those experiments were more variable and inconstant, less comparable, thus not presented here. The cotyledons rendered generally more bands, some of which were less distinct or did not match the leafletbands. The clear cotyledon banding patterns have been independently taken into consideration when discussing the results. Except for a few additional annual strains, additional perennial species of which only cotyledons have been analyzed are: $V$. cracca (No. $327,2 \mathrm{n}=28$ ), V. cassubica (No. $340,2 \mathrm{n}=12$ ), V. truncatula (No. $311,2 \mathrm{n}=$ ?), $\quad V$. sepium (No. 326, $2 \mathrm{n}=14$ ), V. amoena (No. 334, 335, $\mathrm{n}=24$ ), V. unijuga (No. 338, $\mathrm{n}=24$ ).

The study has been carried out during the years 1975-78. 
Table 1. Strains of Vicia species examined in this study

\begin{tabular}{|c|c|c|c|c|c|}
\hline Section & Series & Species & $2 \mathrm{n}$ & Seed source & rain No. \\
\hline \multirow[t]{14}{*}{ Faba } & Fabae & V. bithynia* & 14 & Lehman, 1966 & 129 \\
\hline & & $V$. narbonensis & 14 & Plitmann, 1975 & 306 \\
\hline & & $"$ & 14 & Plitmann, 1975 & 307 \\
\hline & Hyranicae & $V$. hyrcanica & $12(14)$ & Lehman, 1966 & 127 \\
\hline & & V. esdraelonensis & 12 & Plitmann, 1975 & 301 \\
\hline & & $V$. melanops & 10 & Lehman, 1966 & 114 \\
\hline & & " & 10 & Lehman, 1966 & 121 \\
\hline & & $"$ & 10 & Vasileva, 1967 & 144 \\
\hline & Peregrinae & $V$. peregrina & $12 ?$ & Mettin, 1960 & 29 \\
\hline & & " & & Kjellqvist, 1969 & 195 \\
\hline & & " & $14(12)$ & Plitmann, 1975 & 316 \\
\hline & & V. michauxii & 14 & Lehman, 1966 & 128 \\
\hline & & $V$. persepolitana & 14 & Plitmann, 1975 & 305 \\
\hline & Luteae & V. lutea & 14 & Mettin, 1960 & 28 \\
\hline \multirow[t]{12}{*}{ Vicia } & Hybridae & $V$. pannonica & 12 & Plitmann, 1975 & 302 \\
\hline & & V. hybrida & 12 & Mettin, 1960 & 27 \\
\hline & & " & 12 & Vasileva, 1967 & 156 \\
\hline & & $"$ & 12 & Kjellqvist, 1969 & 193 \\
\hline & Sativae & $V$. lathyroides & 12 & Smith, 1975 & 330 \\
\hline & & $V$. sativa** & 12 & Vasileva, 1967 & 161 \\
\hline & & $"$ & 12 & Vasileva, 1967 & 165 \\
\hline & & " & 12 & Moore, 1975 & 332 \\
\hline & & $"$ & 12 & Rousi, 1975 & 325 \\
\hline & & V. amphicarpa & 14 & Stace, 1974 & 337 \\
\hline & & $V$. cordata $^{* *}$ & 10 & Cubero, 1974 & 261 \\
\hline & & $V$. incisa & 14 & Lehman, 1966 & 126 \\
\hline \multirow[t]{4}{*}{ Ervum } & Erviliae & V. ervilia & 14 & Kjellqvist, 1969 & 205 \\
\hline & & $"$ & 14 & Kjellqvist, 1969 & 207 \\
\hline & & $V$. vicioides & $14 ?$ & Vasileva, 1967 & 153 \\
\hline & Hirsutae & $V$. hirsuta & 14 & Fac. Agr. Kagawa Univ., 1960 & 53 \\
\hline \multirow[t]{9}{*}{ Cracca } & Villosae & V. villosa & 14 & Kjellqvist, 1969 & 194 \\
\hline & & " & & Kjellqvist, 1969 & 202 \\
\hline & & $"$ & 14 & Moore, 1975 & 331 \\
\hline & & ssp. dasycarpa & 14 & Shikoku Agr. Exp. sta., 1960 & 54 \\
\hline & & $V$. aff. benghalensis & 14 & Barnard, 1963 & 93 \\
\hline & & $" \prime$ & 14 & Zertova, 1968 & 178 \\
\hline & & $V$. tenuifolia & 24 & Zertova, 1968 & 98 \\
\hline & & $V$. palaestina & 14 & Plitmann, 1975 & 309 \\
\hline & Monanthae & $V$. monantha & 14 & Plitmann, 1975 & 313 \\
\hline
\end{tabular}

* Only a few plants of this strain were obtained.

** The taxa of $V$. sativa-complex should taxonomically be considered as subspecies, though designated here as species. " $V$. persepolitana" is actually a variety of $V$. michauxii. 
Table 2. Methods of isozyme detection

\begin{tabular}{|c|c|c|c|}
\hline Enzymes & Substrate and staining dye & & \\
\hline Amylase & $\begin{array}{l}\mathrm{H}_{2} \mathrm{O} \\
0.02 \mathrm{M} \text { Phosphate buffer pH } 5.7 \\
\text { Soluble starch } \\
\mathrm{I}_{2}-\mathrm{KI}\left(0.1 \% \mathrm{I}_{2} \text { in } 0.5 \% \mathrm{KI} \text { sol acidified with aceticacid }\right)\end{array}$ & $\begin{array}{r}49 \mathrm{ml} \\
50 \mathrm{ml} \\
1 \mathrm{~g}\end{array}$ & $\left\{\begin{array}{l}\text { at } 37^{\circ} \mathrm{C} \\
\text { for } 1 \mathrm{~h}\end{array}\right.$ \\
\hline Esterase & $\begin{array}{l}\mathrm{H}_{2} \mathrm{O} \\
0.12 \mathrm{M} \text { Phosphate buffer } \mathrm{pH} 6.1 \\
1 \% \alpha \text {-Naphthyl acetate } \\
\text { Fast blue RR salt }\end{array}$ & $\left.\begin{array}{r}40 \mathrm{ml} \\
60 \mathrm{ml} \\
2 \mathrm{ml} \\
40 \mathrm{mg}\end{array}\right\}$ & $\left\{\begin{array}{l}\text { at } 37^{\circ} \mathrm{C} \\
\text { for } 45 \mathrm{~min}\end{array}\right.$ \\
\hline GOT* & $\begin{array}{l}0.1 \mathrm{M} \text { Tris- } \mathrm{HCl} \text { buffer } \mathrm{pH} 8 \\
\text { Pyridoxal-5-phosphate } \\
\text { L-aspartic acid } \\
\alpha \text {-Ketoglutaric acid } \\
\text { Fast blue BB salt }\end{array}$ & $\begin{array}{r}100 \mathrm{ml} \\
5 \mathrm{mg} \\
100 \mathrm{mg} \\
100 \mathrm{mg} \\
150 \mathrm{mg}\end{array}$ & $\left\{\begin{array}{l}\text { at } 37^{\circ} \mathrm{C} \\
\text { for } 1 \mathrm{~h}\end{array}\right.$ \\
\hline IPO** &  & $\begin{array}{r}99 \mathrm{ml} \\
1 \mathrm{ml} \\
10 \mathrm{mg} \\
10 \mathrm{mg}\end{array}$ & $\left\{\begin{array}{l}\text { at room temp. } \\
\text { for } 3 \mathrm{~h}\end{array}\right.$ \\
\hline
\end{tabular}

* GOT: Glutamic-oxaloacetic transaminase.

** IPO: Indophenol oxidase.

\section{RESULTS}

Zymograms of amylase, esterase, GOT and IPO showing band patterns of the leaf samples examined, are presented in Fig. 1 to 4 . The species detected were arranged according to their generally recognized systematic order. Considerable variation in enzyme profiles was observed among strains and species. On the other hand, as expected, no particular differences in band patterns were detected among plants of the same strain when individually analyzed. The specific isozyme patterns are as follows:

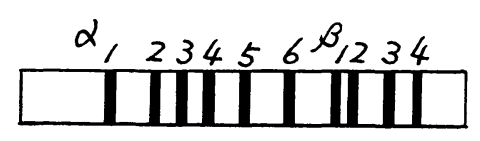

Fig. 1. Total amylase isozyme bands detected in leaf samples of Vicia species.

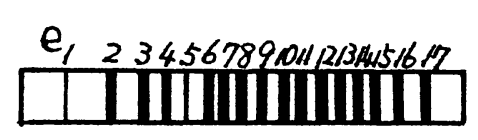

Fig. 2. Total esterase isozyme bands detected in leaf samples of Vicia species. 




Fig. 3. Total GOT isozyme bands detected in leaf samples of Vicia species.



Fig. 4. Total IPO isozyme bands detected in leaf samples of Vicia species.

\section{Amylase band pattern}

The total amylase bands detected are presented in Fig. 1.

The occurrence of each band is indicated in Table 3. As shown in the table, a total of six $\alpha$-amylase and four $\beta$-amylase bands were detected in Vicia strains. $\mathrm{Rf}$ values of $\alpha$-amylase bands were lower than those of $\beta$. A band common to all strains was not observed, but both bands $\alpha_{4}$ and $\beta_{3}$ were detected in most strains and series. Yet series Hyrcanicae and Peregrinae of sect. Vicia show a wider polymorphism in band patterns: Only $\alpha_{2}$ band was detected in $V$. hyrcanica: $\alpha_{2}$ or $\alpha_{3}$ and $\beta_{1}$ or $\beta_{2}$ or $\beta_{3}$ were detected in $V$. melanops and $V$. esdraelonensis. The three strains of $V$. peregrina featured one band of $\alpha\left(\alpha_{1}\right.$ or $\alpha_{2}$ or $\left.\alpha_{4}\right)$ and $\beta_{3}$ band. In $V$. michauxii and $V$. persepolitana of this series both $\alpha_{4}$ and $\beta_{3}$ were present. Two bands, $\alpha_{4}, \beta_{4}$ and $\alpha_{4}, \beta_{3}$, were observed in $V$. lutea and in $V$. hybrida, respectively. In all strains of series Sativae, $\alpha_{4}$ was present but some variation was noticed in the $\beta$-amylase bands: $\beta_{4}$ was detected in $V$. lathyloides, $V$. amphicarpa and $V$. incisa, whereas $\beta_{3}$ was observed in the other taxa. In addition, $\alpha_{6}$ was detected in $V$. amphicarpa and $V$. cordata. In section Ervum, the variation in band patterns was not as wide. In $V$. ervilia and $V$. vicioides, the same bands, $\alpha_{4}$ and $\beta_{3}$, were observed except for one strain of the latter, with $\alpha_{3}$ instead of $\alpha_{4}$, and $V$. hirsuta with $\beta_{4}$ band instead of $\beta_{3}$. In series Villosae of section Cracca, there were in general more bands of both $\alpha$-and $\beta$-amylases compared with other sections. Polymorphism was particularly obvious in $V$. villosa. $V$. monantha has just the $\alpha_{3}$ and $\beta_{3}$ bands whereas in $V$. palaestina $\alpha$-amylase band was absent.

\section{Esterase band pattern}

The occurrence of esterase bands in Vicia species is summarized in Table 4. A total of 17 bands was detected. The number of bands in each strain varied from 4 to 7. Of these, $e_{16}$ band was observed in almost all strains and one or two bands, either $e_{12}$ or $e_{13}$ or $e_{14}$, were common to all strains. In $V$. narbonensis only 4 bands $e_{4}, e_{7}, e_{14}$ and $e_{16}$ were observed. In section Vicia, either $e_{6}$ or $e_{7}$ were detected in all strains. Series Hyrcanicae and Peregrinae show a variation in band pattern similar to that found in amylases. In $V$. lutea only four bands were noticed; 4 or 5 bands were observed in all strains of series Sativae in which some polymorphism was also evident. Species 
Table 3. Occurrence of amylase bands in Vicia species

\begin{tabular}{|c|c|c|c|c|c|c|c|c|c|c|c|}
\hline \multirow{2}{*}{$\begin{array}{c}\text { Strain } \\
\text { No. }\end{array}$} & \multirow{2}{*}{ Species } & \multicolumn{10}{|c|}{ Bands No. } \\
\hline & & $\alpha_{1}$ & 2 & 3 & 4 & 5 & 6 & $\beta_{1}$ & 2 & 3 & 4 \\
\hline $\begin{array}{l}129 \\
306 \\
307\end{array}$ & $\begin{array}{l}V . \text { bithynica } \\
V . \text { narbonensis } \\
\quad " \prime\end{array}$ & & & & + & & & & & $\begin{array}{l}+ \\
+\end{array}$ & \\
\hline $\begin{array}{l}127 \\
301 \\
114 \\
121 \\
144\end{array}$ & $\begin{array}{l}V . \text { hyrcanica } \\
V . \text { esdraelonensis } \\
V . \text { melanops } \\
\quad " \\
\quad "\end{array}$ & & $\begin{array}{l}+ \\
+\end{array}$ & $\begin{array}{l}+ \\
+ \\
+\end{array}$ & & & & + & + & $\begin{array}{l}+ \\
+\end{array}$ & \\
\hline $\begin{array}{r}29 \\
195 \\
316 \\
128 \\
305\end{array}$ & $\begin{array}{c}V . \text { peregrina } \\
" \prime \\
\quad " \\
V . \text { michauxii } \\
V . \text { persepolitana }\end{array}$ & + & + & & $\begin{array}{l}+ \\
+ \\
+\end{array}$ & & & & & $\begin{array}{l}+ \\
+ \\
+ \\
+ \\
+\end{array}$ & \\
\hline 28 & $V$. lutea & & & & + & & & & & & + \\
\hline $\begin{array}{r}302 \\
27 \\
156 \\
193\end{array}$ & $\begin{array}{c}V . \text { pannonica } \\
V . \text { hybrida } \\
\text { " } \\
"\end{array}$ & & & + & $\begin{array}{l}+ \\
+ \\
+\end{array}$ & & & & & $\begin{array}{l}+ \\
+ \\
+ \\
+\end{array}$ & \\
\hline $\begin{array}{l}330 \\
161 \\
165 \\
332 \\
325-1 \\
325-2 \\
337 \\
261 \\
126\end{array}$ & $\begin{array}{c}\text { V. lathyroides } \\
V . \text { sativa } \\
" \prime \\
" \\
" \prime \\
" 1 \\
V . \text { amphicarpa } \\
V . \text { cordata } \\
V . \text { incisa }\end{array}$ & & & & $\begin{array}{l}+ \\
+ \\
+ \\
+ \\
+ \\
+ \\
+ \\
+ \\
+\end{array}$ & & $\begin{array}{l} \\
\\
+ \\
+ \\
+\end{array}$ & & & $\begin{array}{l}+ \\
+ \\
+ \\
+ \\
+ \\
+\end{array}$ & $\begin{array}{l}+ \\
+\end{array}$ \\
\hline $\begin{array}{l}205 \\
207 \\
153-1 \\
153-2\end{array}$ & $\begin{array}{l}V . \text { ervilia } \\
\text { V. vicioides } \\
\text { " }\end{array}$ & & & + & $\begin{array}{l}+ \\
+ \\
+\end{array}$ & & & & & $\begin{array}{l}+ \\
+ \\
+ \\
+\end{array}$ & \\
\hline 53 & $V$. hirsuta & & & & + & & & & & & + \\
\hline $\begin{array}{r}194 \\
202 \\
331 \\
54 \\
93 \\
178 \\
98\end{array}$ & $\begin{array}{l}\text { V. villosa } \\
\quad " \\
\quad " \\
\text { ssp. dasycarpa } \\
V . \text { aff. benghalensis } \\
\quad "{ }^{\prime} \text {. tenuifolia }\end{array}$ & & & $\begin{array}{l}+ \\
+ \\
+\end{array}$ & $\begin{array}{l}+ \\
+ \\
+ \\
+ \\
+ \\
+\end{array}$ & + & & $\begin{array}{l}+ \\
+ \\
+ \\
+ \\
+ \\
+\end{array}$ & $\begin{array}{l}+ \\
+ \\
+ \\
+ \\
+ \\
+\end{array}$ & $\begin{array}{l}+ \\
+\end{array}$ & \\
\hline 309 & $V$. palaestina & & & & & & & & & + & \\
\hline 313 & $V$. monantha & & & + & & & & & & + & \\
\hline
\end{tabular}


Table 4. Occurrence of esterase isozyme bands in Vicia species

\begin{tabular}{|c|c|c|c|c|c|c|c|c|c|c|c|c|c|c|c|c|c|c|}
\hline \multirow{2}{*}{$\begin{array}{l}\text { Strain } \\
\text { No. }\end{array}$} & \multirow{2}{*}{ Species } & \multicolumn{17}{|c|}{ Bands No. } \\
\hline & & $e_{1}$ & 2 & 3 & 4 & 5 & 6 & 7 & 8 & 9 & 10 & 11 & 12 & 13 & 14 & 15 & 16 & 17 \\
\hline 129 & V. bithynica & & & & & & & & & & & & & & & & & \\
\hline 306 & V. narbonensis & & & & + & & & + & & & & & & & + & & + & \\
\hline 307 & " & & & & + & & & + & & & & & & & + & & + & \\
\hline 127 & V. hyrcanica & & + & & + & & & + & & & + & + & & + & & & & \\
\hline 301 & $V$. esdraelonensis & & & & + & & + & & & & + & & & + & & + & + & \\
\hline 114 & $V$. melanops & & + & & + & & & + & & & + & & & & + & & + & \\
\hline 121 & " & & + & & + & & & + & & & + & & & & + & & + & \\
\hline 144 & $\prime \prime$ & + & + & & + & & & + & & & + & & & & + & & + & \\
\hline 29 & $V$. peregrina & & + & & & & & + & & & & + & & + & & + & & \\
\hline 195 & " & & + & & & & & + & & & & + & & + & & & & + \\
\hline 316 & $\prime \prime$ & & + & & & & & + & & & & + & & + & & + & & \\
\hline 128 & V. michauxii & & + & & & & & + & & & & + & & + & & & + & \\
\hline 305 & $V$. persepolitana & & & & + & & & + & & & + & & + & & + & & + & \\
\hline 28 & $V$. lutea & & & & + & & & + & & & & & + & & & & + & \\
\hline 302 & $V \cdot$ pannonica & & & & + & & & + & & & & + & & + & & + & & \\
\hline 27 & V. hybrida & & & + & & & & + & & & & + & & + & & + & & \\
\hline 156 & " & & + & + & & & & + & & & & + & & + & & & + & \\
\hline 193 & $\prime \prime$ & & + & + & & & & + & & & & + & & + & & & + & \\
\hline 330 & $V$. lathyroides & & & & + & & + & & & & & & & + & & & + & \\
\hline 161 & V. sativa & & & & + & & + & & & & & & + & & & & + & \\
\hline 165 & $\prime \prime$ & & + & & & & + & & & & & & & + & & & + & \\
\hline 332 & $\prime \prime$ & & & & + & & + & & & & & & + & + & & & + & \\
\hline $325-1$ & $\prime \prime$ & & & & + & & & + & & & & & & + & & & + & \\
\hline $325-2$ & $" \prime$ & & & & + & & & + & & & & & + & & & & + & \\
\hline 337 & V. amphicarpa & & & & + & & & + & & & & & & + & & & + & \\
\hline 261 & $V$. cordata & & & & + & & + & & & & & & & + & & & + & \\
\hline 126 & $V$. incisa & & & & + & & + & & & & & & & + & & & + & \\
\hline 205 & V. ervilia & & & & + & & & & + & + & & & & + & & & + & \\
\hline 207 & " & & & & + & & & & + & + & & & & + & & & + & \\
\hline $153-1$ & V. vicioides & & & & + & & & & + & + & & & & + & & & + & \\
\hline $153-2$ & " & & & & + & & & & + & + & & & & + & & & + & \\
\hline 53 & $V$. hirsuta & & & & + & + & & & + & + & & & & + & & & + & \\
\hline 194 & V. villosa & & + & & + & & & & + & + & & & & + & & & + & \\
\hline 202 & " & & & + & + & & & & + & + & & & & + & & & + & \\
\hline 331 & $\prime \prime$ & & + & & + & & & & + & + & & & & & + & & + & \\
\hline 54 & ssp. dasycarpa & & & + & + & & & & + & + & & & & & + & & + & \\
\hline 93 & V. aff. benghalensis & & & + & + & & & & + & + & & & & & + & & + & \\
\hline 178 & 11 & & & + & + & & & & + & & & & & & + & & + & \\
\hline 98 & $V$. tenuifolia & & & + & + & & & & + & & & & & & + & + & + & \\
\hline 309 & $V \cdot$ palaestina & & & & & + & + & & & & & & & + & & + & + & \\
\hline 313 & $V$. monantha & & & + & + & & & + & & & & & & + & & & + & \\
\hline
\end{tabular}


Table 5. Occurrence of GOT isozyme bands in Vicia species

\begin{tabular}{|c|c|c|c|c|c|c|c|c|}
\hline \multirow{2}{*}{$\begin{array}{l}\text { Strain } \\
\text { No. }\end{array}$} & \multirow{2}{*}{ Species } & \multicolumn{7}{|c|}{ Bands No. } \\
\hline & & $g_{1}$ & 2 & 3 & 4 & 5 & 6 & 7 \\
\hline $\begin{array}{l}129 \\
306 \\
307\end{array}$ & $\begin{array}{l}V . \text { bithynica } \\
V . \text { narbonensis } \\
\quad " 1\end{array}$ & & $\begin{array}{l}+ \\
+\end{array}$ & & & $\begin{array}{l}+ \\
+\end{array}$ & & \\
\hline $\begin{array}{l}127 \\
301 \\
114 \\
121 \\
144\end{array}$ & $\begin{array}{l}V . \text { hyrcanica } \\
V . \text { esdraelonensis } \\
V . \text { melanops } \\
\quad " \\
\quad "\end{array}$ & & $\begin{array}{l}+ \\
+ \\
+ \\
+ \\
+\end{array}$ & & $\begin{array}{l} \\
+ \\
+ \\
+\end{array}$ & & $\begin{array}{l}+ \\
+\end{array}$ & \\
\hline $\begin{array}{r}29 \\
195 \\
316 \\
128 \\
305\end{array}$ & $\begin{array}{c}V . \text { peregrina } \\
" \prime \\
" \prime \\
V . \text { michauxii } \\
V . \text { persepolitana }\end{array}$ & & $\begin{array}{l}+ \\
+ \\
+ \\
+ \\
+\end{array}$ & $\begin{array}{l}+ \\
+ \\
+ \\
+ \\
+\end{array}$ & & & & \\
\hline 28 & $V$. lutea & & + & & & + & & \\
\hline $\begin{array}{r}302 \\
27 \\
156 \\
193\end{array}$ & $\begin{array}{c}V . \text { pannonica } \\
V . \text { hybrida } \\
\text { " } \\
" 1\end{array}$ & & $\begin{array}{l}+ \\
+ \\
+ \\
+\end{array}$ & & & $\begin{array}{l}+ \\
+ \\
+ \\
+\end{array}$ & & \\
\hline $\begin{array}{l}330 \\
161 \\
165 \\
332 \\
325-1 \\
325-2 \\
337 \\
261 \\
126\end{array}$ & $\begin{array}{c}V . \text { lathyroides } \\
V . \text { sativa } \\
" \prime \\
" \\
" \\
" 1 \\
V . \text { amphicarpa } \\
V . \text { cordata } \\
\text { V. incisa }\end{array}$ & $\begin{array}{l}+ \\
+ \\
+\end{array}$ & $\begin{array}{l}+ \\
+ \\
+ \\
+ \\
+ \\
+ \\
+\end{array}$ & & & $\begin{array}{l}+ \\
+ \\
+ \\
+ \\
+ \\
+ \\
+ \\
+ \\
+\end{array}$ & & \\
\hline $\begin{array}{l}205 \\
207 \\
153-1 \\
153-2\end{array}$ & $\begin{array}{c}V . \text { ervilia } \\
\text { " } \\
V .{ }^{\text {vicioides }} \\
\end{array}$ & & $\begin{array}{l}+ \\
+ \\
+ \\
+\end{array}$ & & & & & $\begin{array}{l}+ \\
+ \\
+ \\
+\end{array}$ \\
\hline 53 & $V$. hirsuta & & + & & & & & + \\
\hline $\begin{array}{r}194 \\
202 \\
331 \\
54 \\
93 \\
178 \\
98\end{array}$ & $\begin{array}{l}\text { V. villosa } \\
" \prime \\
" \prime \\
\text { ssp. dasycarpa } \\
\text { V. aff. benghalensis } \\
\text { " } \\
\text { V. tenuifolia }\end{array}$ & & $\begin{array}{l}+ \\
+ \\
+ \\
+ \\
+ \\
+ \\
+\end{array}$ & & & $\begin{array}{l}+ \\
+ \\
+\end{array}$ & $\begin{array}{l}+ \\
+\end{array}$ & \\
\hline 309 & $V$. palaestina & & + & & & + & & \\
\hline 313 & $V$. monantha & & + & & & + & & \\
\hline
\end{tabular}


Table 6. Occurrence of IPO isozyme in Vicia species

\begin{tabular}{|c|c|c|c|c|c|c|c|c|c|c|}
\hline \multirow{2}{*}{$\begin{array}{l}\text { Strain } \\
\text { No. }\end{array}$} & \multirow{2}{*}{ Species } & \multicolumn{9}{|c|}{ Bands No. } \\
\hline & & $i_{1}$ & 2 & 3 & 4 & 5 & 6 & 7 & 8 & 9 \\
\hline 129 & $V$. bithynica & + & + & + & & + & & + & + & \\
\hline $\begin{array}{l}306 \\
307\end{array}$ & $\begin{array}{c}\text { V. narbonensis } \\
"\end{array}$ & $\begin{array}{l}+ \\
+\end{array}$ & $\begin{array}{l}+ \\
+\end{array}$ & $\begin{array}{l}+ \\
+\end{array}$ & & $\begin{array}{l}+ \\
+\end{array}$ & & $\begin{array}{l}+ \\
+\end{array}$ & $\begin{array}{l}+ \\
+\end{array}$ & \\
\hline 127 & $V$. hyrcanica & + & + & + & + & + & & + & & + \\
\hline 301 & $V$. esdraelonensis & + & + & + & & + & & + & & + \\
\hline 114 & $V$. melanops & + & + & + & + & + & & + & + & \\
\hline $\begin{array}{l}121 \\
144\end{array}$ & " & $\begin{array}{l}+ \\
+\end{array}$ & $\stackrel{+}{+}$ & $\begin{array}{l}+ \\
+\end{array}$ & $\begin{array}{l}+ \\
+\end{array}$ & $\begin{array}{l}+ \\
+\end{array}$ & & $\begin{array}{l}+ \\
+\end{array}$ & $\begin{array}{l}+ \\
+\end{array}$ & \\
\hline 29 & $V$. peregrina & + & + & + & + & + & & + & & \\
\hline 195 & " pol & + & + & + & + & + & & + & + & \\
\hline 316 & & + & + & + & + & + & & + & + & \\
\hline $\begin{array}{l}128 \\
305\end{array}$ & $\begin{array}{l}V . \text { michauxii } \\
V . \text { persepolitana }\end{array}$ & $\begin{array}{l}+ \\
+\end{array}$ & $\begin{array}{l}+ \\
+\end{array}$ & $\begin{array}{l}+ \\
+\end{array}$ & $\begin{array}{l}+ \\
+\end{array}$ & $\begin{array}{l}+ \\
+\end{array}$ & & $\begin{array}{l}+ \\
+\end{array}$ & + & + \\
\hline 28 & $V$. lutea & + & + & + & & + & & + & + & \\
\hline 302 & $V \cdot$ pannonica & + & + & + & & + & & + & & + \\
\hline 27 & $V$. hybrida & + & + & + & & + & & + & & + \\
\hline $\begin{array}{l}156 \\
193\end{array}$ & " & $\begin{array}{l}+ \\
+\end{array}$ & $\begin{array}{l}+ \\
+\end{array}$ & $\stackrel{+}{+}$ & & $\begin{array}{l}+ \\
+\end{array}$ & & $\begin{array}{l}+ \\
+\end{array}$ & & $\begin{array}{l}+ \\
+\end{array}$ \\
\hline 330 & V. lathyroides & + & + & + & & + & & + & & \\
\hline 161 & $V$. sativa & + & + & + & & + & + & + & & \\
\hline $16 \overline{5}$ & " & + & + & + & & + & + & + & & \\
\hline 332 & $"$ & + & + & + & & + & + & + & & \\
\hline $325-1$ & $"$ & + & + & + & & + & + & + & & \\
\hline $325-2$ & & + & + & + & & + & + & + & & \\
\hline 337 & V. amphicarpa & $\stackrel{+}{+}$ & $\stackrel{+}{+}$ & $\begin{array}{l}+ \\
+\end{array}$ & & $\stackrel{+}{+}$ & $\begin{array}{lll}+ \\
+\end{array}$ & $\stackrel{+}{+}$ & & \\
\hline $\begin{array}{l}261 \\
126\end{array}$ & $\begin{array}{l}V . \text { cordata } \\
V . \text { incisa }\end{array}$ & $\stackrel{+}{+}$ & $\begin{array}{l}+ \\
+\end{array}$ & $\begin{array}{l}+ \\
+\end{array}$ & & $\begin{array}{l}+ \\
+\end{array}$ & $\begin{array}{l}+ \\
+\end{array}$ & $\begin{array}{l}+ \\
+\end{array}$ & & \\
\hline 205 & $V$. ervilia & + & + & + & & + & & + & + & \\
\hline & & + & + & + & & + & & + & + & \\
\hline $\begin{array}{l}153-1 \\
153-2\end{array}$ & $\begin{array}{l}\text { V. vicioides } \\
\prime \prime\end{array}$ & $\begin{array}{l}+ \\
+\end{array}$ & $\begin{array}{l}+ \\
+\end{array}$ & $\begin{array}{l}+ \\
+\end{array}$ & & $\begin{array}{l}+ \\
+\end{array}$ & & $\begin{array}{l}+ \\
+\end{array}$ & $\begin{array}{l}+ \\
+\end{array}$ & \\
\hline 53 & $V$. hirsuta & + & + & + & & + & + & + & + & \\
\hline & & + & + & + & & + & & 1 & 1 & \\
\hline $\begin{array}{l}194 \\
202\end{array}$ & $\begin{array}{c}V . \text { villosa } \\
\prime \prime\end{array}$ & + & $\begin{array}{l}+ \\
+\end{array}$ & + & & $\begin{array}{l}+ \\
+\end{array}$ & & $\begin{array}{l}+ \\
+\end{array}$ & $\begin{array}{llll}+ \\
+\end{array}$ & \\
\hline 331 & $"$ & + & + & + & & + & & + & + & \\
\hline 54 & ssp. dasycarpa & + & + & + & & + & & + & + & \\
\hline 93 & V.aff. benghalensis & + & + & $t$ & & + & & + & + & \\
\hline 178 & ". as & + & + & + & & + & & + & + & \\
\hline 98 & $V$. tenuifolia & + & + & + & & + & & + & + & \\
\hline 309 & V. palaestina & + & + & + & & + & & + & + & \\
\hline 313 & $V$. monantha & + & + & + & & + & & + & + & \\
\hline
\end{tabular}


of section Ervum have the same pattern $\left(e_{4}, e_{8}, e_{9}, e_{13}\right.$ and $\left.e_{16}\right)$, with $e_{5}$ in $V$. hirsuta, additional to this pattern. In section Cracca there were averagely more bands and only $e_{16}$ was common to all strains. Difference in band pattern (and number) was observed between the two morphological groups, one of which is $V$. villosa group including ssp. dasycarpa, $V$. benghalensis and $V$. tenuifolia, the other represented by $V$. palaestina. In this respect, $V$. esdraelonensis is as similar to the latter species as to members of Hyrcanicae.

\section{GOT band pattern}

The occurrence of GOT isozyme bands is summarized in Table 5. A total of seven bands was detected. In each strain two bands were observed. The $g_{2}$ band was detected in almost all strains; however, in two strains of $V$. sativa complex, $g_{1}$ band was observed instead of $g_{2}$. Certain taxonomic entities could be characterized by the $\mathrm{Rf}$ values of GOT bands other than $g_{2}$. For example, band $g_{6}$ was detected in $V$. hyrcanica and $V$. esdraelonensis, band $g_{4}$ in $V$. melanops. Strains of series Peregrinae have typically $g_{3}$ band; Luteae, Hybridae and Sativae have $g_{5}$ bands. In section Ervum, all strains have $g_{7}$ band; in section Cracca either $g_{5}$ or $g_{6}$ bands were detected. The latter section, and to some extent $V$. sativa complex (i. e., including all related taxa) were the more polymorphic.

\section{IPO band pattern}

The occurrence of IPO bands is summarized in Table 6. A total of nine bands was detected; in each species or strain five to seven bands were observed. Of these, five bands $\left(i_{1}, i_{2}, i_{3}, i_{5}\right.$ and $\left.i_{7}\right)$ were common to all strains. Other bands are characteristic to certain taxonomic entities, and might be indicative to either differences or similarities between those. In all strains of series Fabae, Erviliae, Hirsutae and Villosae, $i_{8}$ band was present, $i_{9}$ missing. In series Hyrcanicae and the related Peregrinae, $i_{4}$ band was detected. Band $i_{6}$ was observed in all strains of series Sativae, except $V$. lathyloides, also in $V$. hirsuta of section Ervum. Band $i_{9}$ was detected in V. hyrcanica, $V$. esdraelonensis, $V$. persepolitana, $V$. hybrida and $V$. pannonica instead of $i_{8}$ band. In accordance with the slight difference in $\mathrm{Rf}$ values between $i_{8}$ and $i_{9}$ bands, which substitute one another, it is presumed that both $i_{8}$ and $i_{9}$ represent two complementary alleles (as is the case of $\beta_{3}$ and $\beta_{4}$ amylase bands).

\section{DISCUSSION}

During the last decade many reports on isozyme polymorphism with bearings on taxonomic relationships have been published. Many of these works are dealing with cultivated plants and their wild relatives. Such are studies on rice cultivars (Shahi et al. 1969; Nakagawara 1977), Carthamus (Efron et al. 1973), Chrysanthemum (Krisper and Puff 1975), tomatoes (Rick and Fobes 1975), Brassica (Denford 1976; Denford and Vaughan 1977), Citrus (Esen and Scora 1977) and soybean (Broué et al. 1977), not to mention the many studies of this kind on wheat and barley. Most, if not all, add electrophoretic data to the taxonomic evaluation, either as additional diagnostic criteria for identification of species and strains, or in order to confirm or establish presumed systematic 
relationships (and refer to Boulter et al. 1966; Avise 1974). Electrophoretic studies in Vicia have so far been carried out on proteins in general (e. g. Ladizinsky 1975; Perrino et al. 1977), or limited to special groups (Yamamoto 1975, 1979; Bassiri and Adams 1978).

From the present study, albeit encompassing only representative species and 4-5 enzyme systems, some indications as to specific taxonomic resemblances or distinctions and systematic affinities could already be drawn. The value of isozyme patterns in the taxonomy and evolution of Vicia could also be generally considered.

Certain bands $\left(i_{1}, i_{2}, i_{3}, i_{5}, i_{7}\right)$ were practically common to all sections, some others $\left(\alpha_{4}, \beta_{3}, e_{4}, e_{16}, g_{2}, i_{8}\right)$ to most series. Four pairs were complementary bands, usually with very close $\mathrm{Rf}$ values: $\beta_{3}$ and $\beta_{4}, e_{16}$ and $e_{17}, g_{1}$ and $g_{2}, i_{8}$ and $i_{9}$. Presence or absence of the remaining bands was sometimes a feature typical to sections, series even species, thus helpful in taxonomic identification. For instance, $\alpha_{3}$ and $\alpha_{4}$ are together highly frequent in section Cracca, but $\alpha_{3}$ appears just occasionally in other sections; $\beta_{1}$ and $\beta_{2}$ are quite common in series Villosae (characterized also by usually 4 amylase bands), $\beta_{3}$ in the rest. Note that $\alpha_{2}$ and $\alpha_{3}, \beta_{1}, \beta_{2}$ and $\beta_{4}$ feature genetic polymorphism in most series. No $\alpha$ - or $\beta$-amylase bands could be detected in $V$. palaestina and $V$. hyrcanica, respectively. Further, $e_{4}$ is typically absent in some species mainly of section Vicia, $e_{7}$ is most common in series of this section other than Sativae, $e_{8}$ and $e_{9}$ typical of series Villosae, both and $e_{13}$ of section Ervum. Polymorphism is particularly shown for bands $e_{2}$ to $e_{4}, e_{11}$ and $e_{12}$ in section Vicia, $e_{13}$ to $e_{15}$ in section Cracca. The advanced series Luteae, Sativae and probably Fabae are characterized by only 4 (instead of $5-6$ ) esterase bands.

Band $g_{5}$ is quite common in section Cracca, and certain series of section Vicia, but absent in section Ervum (characterized by $g_{7}$ ) and the inter-related series Hyrcanicae and Peregrinae. Band $i_{4}$ is typical of series Hyrcanicae and Peregrinae. On the other hand, $i_{8}$ and $i_{9}$ are missing in series Sativae.

Some uncommon bands, like $\alpha_{1}, \alpha_{6}, e_{1}, i_{4}$ or $i_{6}$ might be considered as new additions to the common patterns in series of section Vicia (and referred to Endo 1973).

In general, there are common vs. differentiating specific bands, yet their systematic distribution is quite haphazard and there are not overall enzymatic patterns characterizing categories higher than species.

Some systems, however, (like GOT or $\mathrm{ADH}$ ), which are less polymorphic on intraspecific level, might be useful for taxonomic delimitation of certain species, series or sections, whereas the others (amylase, esterase) might be helpful in defining intraspecific entities.

Association coefficients have been calculated from both leaflet and cotyledon analysis. The overall similarity between strains of same species might range from 0.5 to 0.85. The similarity between species of the same group is high, but sometimes lower. Yet, partial results show that $\mathrm{ADH}$ and GOT are more homogenous, with many high coefficients for all species; dissimilarities when indicated are usually on higher taxonomic levels. IPO, $\alpha$-esterase even amylase are more heterogenic in this sense, hence more useful for specific (or lower categories) delimitation. The resemblances between Vicia species, evaluated according to similarities in isozyme patterns are, again, irregular 
in distribution, the enzymatic similarity does not necessarily correspond with the systematic affinity except for certain cases.

Thus, series Hybridae and Luteae considered systematically closely related show a high degree of resemblance in their GOT, amylase and IPO patterns, which is also in accordance with karyotypic similarity reported by Yamamoto (1973). They are also more associated with series Sativae than with any other group. Few associations of this kind could also be traced between species of a certain group. Accordingly, the subdivision of series Villosae is reconfirmed by amylase and esterase patterns; $V$. esdraelonensis is more related by isozyme resemblance to $V$. hyrcanica than to any other species; $V$. michauxii and $V$. persepolitana are very similar and represent two entities (populations) of a polymorphic taxon.

However, the more diverse are the taxonomic entities-the more isozyme polymorphism and the less similarity are observed.

It is quite clear that in polymorphic species or complexes-there is also more pronounced polymorphism in banding patterns. These results are compatible with the morphological, ecological and karyotypic patterns in those groups (referred to Zohary and Plitmann 1978). It is no wonder, therefore, that differences mainly in amylase and esterase systems, between strains of $V$. sativa, between strains and species of series Villosae and Peregrinae were more noticeable than in other groups. Those are probably the most variable and polymorphic taxa in the genus. Some of those are colonizers and the uniformity within and variation between populations or races, conform the situation described in Xanthium (Moran and Marshall 1978).

On the other hand, section Ervum, presumably the more primitive (according to morphology and karyotypes) is quite unique and probably the less polymorphic in isozyme patterns, which corresponds with its relative morphological homogeneity.

No direct correlation with particular morphological characters, such as colour of flower, habitus (the new perennials versus the annuals) and pubescence, was so far noticed. Bands $\alpha_{3}$ and $e_{4}$ were significantly common in species with distinct peduncles; $e_{8}$ and $e_{9}$ (typical of section Ervum and series Villosae) were absent in the nonpeduncled species, concurrently rare in species with $2 \mathrm{n}=12$. Likewise, $i_{8}$ was common in the $2 \mathrm{n}$ $=14$ species (sections Cracca and Ervum), rare in the others. Except for the above, no further correlations with chromosome numbers could be traced.

Another aspect of interest is the evolutionary implication of the isozyme variability in Vicia. At this stage we cannot point to any definite biochemical trends with phylogenetic significance. Yet, there are clear indications that certain systems are rather stable and uniform (or of very low selection rate)-whereas others are more variable, showing higher polymorphism. It might correspond with their different metabolic functions referred to Johnson (1971 and 1974).

At any rate, enzymatic polymorphism is more expressed in taxa polymorphic also in other characters. These vetches have wide and diverse ecological range and they are commonly selfers with occasional outcrossing. The actual selective value, if any, of this enzymatic polymorphism is still a matter of debate. No doubt, the present-day situation in Vicia reflects a complex array of evolutionary processes such as ancient divergence and recent genetic change (either 'neutral' or through direct selection), 
together with the influence of biological and environmental factors, differing in different species.

The present study, though preliminary, might serve as a basis for further investigations along various lines: Detailed comparisons between species of the Old and New World in order to find out whether their evolution was parallel, or not; Correlations between habitus (and other morpho-anatomical criteria) and isozyme patterns, which might help in the taxonomy of certain groups, like section Cracca. Likewise, correlations between isozyme polymorphism and the breeding system (by which it might be possible to measure the amount of cross-pollination of Vicia species, many of which are predominantly selfers), and, even more, between such polymorphism and the ecological situation (particularly to find out the impact of disturbed and secondary habitats); Analysis of other enzyme systems, also of additional species, in order to significantly calculate genetic distances and thus to reach a better understanding of the phylogenetic relationships and evolution in Vicia.

\section{ACKNOWLEDGMENTS}

This study was kindly supported by the Japan Society of Promotion of Sciences (JSPS). Thanks are due to people and institutions who supplied us with seed accessions and to the many students who helped in work.

\section{LITERATURE CITED}

Avise, J. C., 1974 Systematic value of electrophoretic data. Syst. Zool. 23: 465-481.

Bassiri, A., and M. W. Adams, 1978 Evaluation of common bean cultivar relationships by means of isozyme electrophoretic patterns. Euphytica 27: 707-720.

Boulter, D., D. A. Thurman, and B.L. Turner, 1966 The use of disc electrophoresis of plant proteins in systematics. Taxon 15: 135-143.

Brewer, P. J., 1970 "An Introduction to Isozyme Techniques". Academic Press, New York.

Broué, P., D. R. Marshall, and W. J. Müller, 1977 Biosystematics of subgenus Glycine (Verdc.): Isoenzymatic data. Austr. J. Bot. 25: 555-566.

Davis, B. J., 1964 Disc electrophoresis. II. Method and application to human serum proteins. Ann. New York Acad. Sci. 121: 404-427.

Denford, K. E., 1976 Isoenzyme studies in members of the genus Brassica. Bot. Notiser 128: 455-462.

Denford, K.E., and J.G. Vaughan, 1977 A comparative study of certain seed isoenzymes in the ten chromosome complex of Brassica campestris. Ann. Bot. 41: 411-418.

Efron, Y., M. Peleg, and A. Ashri, 1973 Alcohol dehydrogenase allozymes in the safflower genus Carthamus L. Biochem. Genet. 9: 299-308.

Endo, T., 1973 Isozyme loci and a strategy of differentiation in plants A review. Seiken Ziho 24: 89-104.

Esen, A., and R. W. Scora, 1977 Amylase polymorphism in Citrus and some related genera. Amer. J. Bot. 64: 305-309.

Frydenberg, O., and G. Nielsen 1965 Amylase isozymes in germinating barley seeds. Hereditas 54: $123-139$.

Hollings, E., and C. A. Stace, 1974 Karyotype variation and evolution in the Vicia sativa aggregate. New Phytol. 73: 195-208.

Johnson, G. B., 1971 Metabolic implications of polymorphism as an adaptive strategy. Nature 
232: $347-349$.

Johnson, G. B., 1974 Enzyme polymorphism and metabolism. Science 184: 28-37.

Johnson, G. B., 1975 Enzyme polymorphism and adaptation. Stadler Symp. 7: 91-116.

Krisper, J., and C. Puff, 1976 Peroxidasemuster und Systematik von Argyrantherum und Chrysanthemum s. str. (Asteraceae-Anthemideae). P1. Syst. Evol. 124: 291-301.

Kupicha, F. K., 1976 The infrageneric structure of Vicia. Notes Roy. Bot. Gard. Edinb. 34: 283-326.

Ladizinsky, G., 1975 Seed protein electrophoresis of the wild and cultivated species of section Faba of Vicia. Euphytica 24: 785-788.

Ladizinsky, G., 1978 Chromosomal polymorphism in wild populations of Vicia sativa L. Caryologia 31: 233-241.

Moran, G. F., and D. R. Marshall, 1978 Allozyme uniformity within and variation between races of the colonizing species Xanthium strumarium L. Austr. J. Biol. Sci. 31: 283-291.

Nakagawara, M., 1977 Genetic analysis for esterase isoenzymes in rice cultivars. Japan J. Breed. 27: $141-148$.

Perrino, P., I. Maellaro, and A. Blanco, 1977 Patterns di electroforesi in specie del genere Vicia. Genet. Agrar. 31: 121-130.

Phelan, J. R., and J.G. Vaughan, 1976 Study of Brassica oleracea with particular reference to its relationship to Brassica alboglabra. Bioch. Syst. Eco. 4: 173-178.

Plitmann, U., 1970 Vicia L (annuals). In "Flora of Turkey" (P. H. Davis, ed.) vol. 3. Edinburgh Univ. Press., Edinburgh.

Rick, Ch. M., and J. F. Fobes, 1975 Allozyme variation in the cultivated tomato and closely related species. Bull. Torrey Bot. Club 102: 376-384.

Shahi, B. B., H. Morishima, and H. I. Oka, 1969 A survey of variations in peroxidase, acid phosphatase and esterase isozymes of wild and cultivated Oryza species. Japan. J. Genetics 44: 303-319.

Yamamoto, K., 1973 Karyotaxonomical studies on Vicia. I. On the karyotype and character of some annual species of Vicia. Japan. J. Genetics 48: 315-327.

Yamamoto, K., 1975 Estimation of genetic homogeneity by isozymes from interspecific hybrids of Vicia. I. Japan. J. Breed. 25: 60-64.

Yamamoto, K., 1979 Estimation of genetic homogeneity by isozymes from interspecific hybrids of Vicia. II. Japan. J. Breed. 29: 59-65.

Zohary, D., and U. Plitmann, 1979 Chromosome polymorphism, hybridization and colonization in the Vicia sativa group (Fabaceae). P1. Syst. Evol. 131: 143-156.

Zauros, E., 1975 Electrophoretic variation in allozymes related to function or structure? Nature 254: $446-448$. 